\title{
RASTREIO DE MEMÓRIA EM IDOSOS COM E SEM DOENÇAS CRÔNICAS NÃO TRANSMISSÍVEIS
}

\author{
MEMORY SCREENING IN ELDERLY WITH AND WITHOUT NON-COMMUNICABLE \\ CHRONIC DISEASES
}

\section{RESUMO}

O presente estudo objetivou observar o desempenho de idosos com e sem doenças crônicas não transmissíveis em provas de rastreio de memória. Para isso, foi realizado um estudo de campo, descritivo, observacional e transversal realizado em unidade de saúde da família e em um projeto social para idosos de uma capital nordestina. Participaram da pesquisa 65 idosos, de ambos os gêneros, com idades variando entre 60 e 87 anos, que aceitaram participar da pesquisa mediante a assinatura do termo de Consentimento Livre e Esclarecido e autorização dos respectivos locais. Para realização da pesquisa foi utilizada a Bateria Breve de Rastreio Cognitivo. Os dados foram analisados quantitativamente, a partir de estatística descritiva e inferencial pelo teste de correlação de Pearson, com nível de significância de 5\%. A maioria dos idosos $(64,61 \%)$ declarou ter queixa de memória e declarou não ter queixa de linguagem. Foi observado que os idosos sem doenças crônicas não transmissíveis apresentaram o melhor desempenho nos testes de memória, enquanto os idosos com diabetes mellitus apresentaram o pior desempenho. Verificou-se correlação negativa entre a idade e o desempenho nas provas de memória imediata $\left(p=0,004^{*}\right)$, Aprendizado $\left(p=0,02^{*}\right)$, Memória Tardia $\left(p=0,013^{*}\right)$ e pela quantidade de Intrusões realizadas $\left(p=0,006^{*}\right)$. Com isso, foi observado que o declínio cognitivo de memória em idosos aumenta de acordo com o avanço da idade. A maioria dos idosos possui queixa de memória. E a diabetes mellitus foi a doença crônica com pior desempenho nos testes de memória.

Palavras-chave: Idoso. Memória. Doenças crônicas. Saúde Pública. Fonoaudiologia.

\footnotetext{
1 Mestre em Linguística pela Universidade Federal da Paraíba (UFPB). Docente do Centro Universitário de João Pessoa (UNIPÊ) E-mail: ivonaldo.lima@unipe.br

2 Graduada em Fonoaudiologia pelo Centro Universitário de João Pessoa (UNIPÊ). E-mail: anapaulaac08@gmail.com 3 Graduanda em Fonoaudiologia pelo Centro Universitário de João Pessoa (UNIPÊ). E-mail: alanaemilyy@hotmail.com 4 Graduanda em Fonoaudiologia pelo Centro Universitário de João Pessoa (UNIPÊ). E-mail: fernandacardozol3@gmail.com 5 Graduada em Fonoaudiologia pelo Centro Universitário de João Pessoa (UNIPÊ). E-mail: ggilcelia71@gmail.com 6 Graduada em Fonoaudiologia pelo Centro Universitário de João Pessoa (UNIPÊ). E-mail: nahfono@gmail.com
} 


\section{ABSTRACT}

The present study aimed to observe the performance in memory screening tests of elderly with and without non-communicable chronic diseases. For this, we were realized a descriptive, observational and cross-sectional field study carried out at the family health unit and at a social project in the northeastern capital. A total of 65 elderly people, both genders, aged between 60 and 87 years, who agreed to participate in the research, signed the term of Free and Informed Consent and authorization of the respective places. The Cognitive Screening Brief Battery was used to perform the research. Data were analyzed quantitatively from descriptive and inferential statistics using the Pearson correlation test, with a significance level of $5 \%$. The majority of the elderly $(64.61 \%)$ reported having a memory complaint and declared that they had no language complaint. It was observed that the elderly without noncommunicable diseases presented the best performance in the memory tests, while the elderly with diabetes mellitus presented the worst performance. There was a negative correlation between age and performance in the immediate memory tests $\left(p=0.004^{*}\right)$, Learning $\left(p=0.02^{*}\right)$, Late Memory $\left(p=0.013^{*}\right)$ and the number of Intrusions performed $\left(p=0.006^{*}\right)$. Conclusion: It was observed that the cognitive decline of memory in the elderly increases with the advancement of age. Most of the elderly have memory complaints. And diabetes mellitus was the chronic disease with the worst performance in memory tests.

Keywords: Aged. Memory. Noncommunicable diseases. Public health. SpeechLanguage and Hearing Sciences.

\section{INTRODUÇÃO}

Brasil possui a quinta maior população idosa do mundo, com 28 milhões de idosos com 60 anos ou mais. Conforme análise da Fundação Oswaldo Cruz, através dos dados da Pesquisa Nacional de Saúde (PNS 2013), um em cada três idosos brasileiros apresentaram alguma restrição funcional. Destes, $80 \%$, cerca de 6,5 milhões de idosos, conta com auxílio de familiares para realizar alguma atividade do cotidiano, mas 360 mil não contêm esse apoio. A falta de condições física e mentais causa sofrimento para o idoso e para aos familiares (BRASIL, 2016).

No transcorrer do processo de envelhecimento é natural algumas funções cognitivas se diminuam, como a atenção, as funções executivas e a memória, havendo a lentificação no processamento das informações. A integralidade da função cognitiva em idosos colabora para a autonomia e 
a capacidade de autocuidado. O declínio das habilidades cognitivas tornase um fator associado ao aumento do risco de dificuldades no desempenho de atividades instrumentais de vida diária e até dificuldades no convívio social. Entre os déficits cognitivos, tem-se aqueles que envolvem a memória é caracterizado como queixa mais frequente em idosos (BERNARDES et al, 2016; CAMPOLINA 2013). Portanto, o declínio cognitivo é uma das consequências do envelhecimento mais temida pelos idosos, sendo necessário investigar fatores de risco associados a esse declínio (BERNARDES et al., 2016).

O aumento demográfico da população acima de 60 anos provocou alterações nos padrões de saúde. Houve uma diminuição da morbidade e mortalidade por doenças infecciosas, enquanto houve um aumento das doenças crônicas não transmissíveis, como diabetes mellitus (DM) e hipertensão arterial sistêmica (HAS), têm sido registradas como os principais motivos de morte e incapacidades entre idosos (CEMBRANEL et al., 2017; CAMPOLINA, DINI, CICONELLI, 2011).

O envelhecimento combinado a uma doença, como o diabetes mellitus, traz ao indivíduo, prejuízos ainda maiores (ALVARENGA, PEREIRA, ANJOS, 2010; ARAKI, ITO, 2009; MAGO et al., 2007). O DM é uma síndrome de etiologia múltipla, decorrente da ausência de insulina e/ou da inabilidade do hormônio desempenhar adequadamente seus efeitos, podendo levar ao aparecimento de doenças associadas, como retinopatia, nefropatia, neuropatia periférica, falta de mobilidade articular e força muscular. Além de prejuízo na função cognitiva dos indivíduos. Idosos com diabetes mellitus tipo 2 são mais predispostos a apresentarem algum déficit cognitivo quando colacionados àqueles sem a doença (ALVARENGA, PEREIRA, ANJOS, 2010; RYAN, GEKCLE, 2000).

As mudanças estruturais e funcionais no sistema nervoso central (SNC) do processo do envelhecimento, como nas estruturas corticais e subcorticais, provocam alterações nas funções neurocognitivas dos idosos devido às modificações no metabolismo. Há evidências que déficits da aprendizagem e da memória podem ser resultado de uma influência mútua entre as alterações do metabolismo relacionadas ao diabetes, em que as alterações na concentração da glicose sanguínea afetam a função cerebral, e as modificações estruturais e funcionais que ocorrem no SNC, decorrentes do processo habitual do envelhecimento (ALVARENGA, PEREIRA, ANJOS, 2010; ARAKI, ITO, 2009).

Contudo, esses déficits cognitivos possivelmente se limitam aos processos mais complexos, relacionados ao lobo frontal, como a função executiva, que está relacionada à habilidade no planejamento de estratégias de resolução de problemas e execução de metas (ALVARENGA, PEREIRA, ANJOS, 2010; MUNSHI et al., 2006). 
Já os idosos hipertensos apresentam um risco maior de declínio cognitivo como a lentidão nas respostas, memória e função executiva (CEMBRANEL, 2017). A HAS é considerada um fator de risco para diversas doenças, tais como insuficiência coronariana, acidente vascular cerebral e insuficiência renal que estão associadas a alterações do desempenho cognitivo (MATOSO et al., 2013; MURRAY et al., 2002).

A memória envolve o processo de codificação armazenamento e recuperação das informações, que podem ser alteradas de acordo com a experiências pregressas do indivíduo. E esse sistema podem ser classificados como tipo natureza - explícita, implícita ou operacional/trabalho, ou pelo tempo limite de manutenção dessa informação (curto e longo prazo) (SILVA et al., 2017).

As queixas de alteração de memória em idosos estão presentes em mais de $50 \%$ dessa população. As causas podem variar desde uma percepção de lapsos de memória, efeito de medicação e doenças sistêmicas, abuso de álcool e depressão, até a síndrome demencial em que o comprometimento da memória e de outra área cognitiva (linguagem, praxias, orientação, função executiva, entre outras (SILVA et al., 2017; NITRINI et al., 2005).

No processo de envelhecimento, doenças crônicas não transmissíveis são frequentemente encontradas entre os idosos. Assim, estas são fatores de risco para o declínio cognitivo nesta população, podendo afetar sua capacidade funcional e envelhecimento ativo. Diante disso, esse estudo objetivou observar o desempenho de idosos com e sem doenças crônicas não transmissíveis em provas de rastreio de memória.

\section{FUNDAMENTAÇÃO TEÓRICA}

O envelhecimento da população se tornou um fenômeno mundial iniciado, a princípio, nos países desenvolvidos, na década de 1940 e 1950. Conforme a Organização Mundial de Saúde (OMS), existem atualmente no mundo 600 milhões de pessoas com idade superior a 60 anos. Segundo as projeções dessa entidade, esse contingente populacional deve duplicar em 2025, atingindo 1.200.000.000 de pessoas em todo o Planeta (MAC-KAY, 2011).

O envelhecimento é um processo dinâmico e progressivo que faz parte do ciclo vital. No aspecto biológico, limita a capacidade física e orgânica do indivíduo e o papel desempenhado por ele no meio sociocultural, interferindo diretamente nas suas condições de autonomia e inde-pendência (ABREU, FORLENZA, BARROS, 2005).

Diante de todos os aspectos de mudança física e social que surgem na vida do idoso, a comunicação também constitui um fator decisivo para o bem-estar dos mesmos, pois durante o envelhecimento natural, as habilidades sensoriais, motoras, 
cognitivas e linguísticas envolvidas sofrem modificações. Diante desses aspectos, torna-se evidente a necessidade de intervenções efetivas nos aspectos linguísticoscognitivos, buscando habilitar essa comunicação em seus mais diversos aspectos, entre eles a fala. Esta, por sua vez, passa por adaptações quando se depara com mudanças e transformações socioculturais e/ou anatomo-funcionais, como ocorre nas doenças crônicas não transmissíveis.

Em meio a essa nova configuração populacional do nosso país, tornase uma responsabilidade direta das políticas públicas de saúde e educação a organização rápida e eficiente para fazer face às novas demandas. Outro desafio que se apresenta é a criação de oportunidades para que os avanços da ciência e da tecnologia sejam aplicados de tal forma que o ser humano alcance limites maiores de qualidade de vida independente, produtiva e saudável.

Esses dados reforçam a necessidade de se repensar como o idoso pode ter uma qualidade de vida satisfatória, aumentar a sobrevida e exercer suas atividades quer sejam na vida profissional ou no seu dia-a-dia, sem maiores prejuízos. Isso pode ser atribuído a um estímulo de vida mais saudável, mudanças simples no seu cotidiano, através da diminuição dos fatores de risco tais como, alimentação inadequada, tabagismo, obesidade e sedentarismo que levam as doenças comprometendo cada vez mais a saúde desse idoso.

Contudo, a prevalência de doenças crônicas não transmissíveis eleva-se a partir dos 60 anos, destacando-se: as doenças osteoarticulares, a hipertensão arterial sistêmica (HAS), as doenças cardiovasculares, o diabetes mellitus, as doenças respiratórias crônicas, a doença cerebrovascular e o câncer. Dados nacionais apontam que as DCNT respondem por 66,3\% da carga de doença, enquanto as doenças infecciosas, por 23,5\%, e causas externas, por 10,2\% (CAMPOLINA et al, 2013).

O processo de envelhecimento saudável acarreta modificações heterogêneas nas habilidades de comunicação dos idosos, decorrentes da constituição genética, história de vida, condições de saúde, processos biológicos e neurocognitivos, ocupação/profissão e estruturas socioeconômicas e cultural (MAC-KAY, 2011). A presença de doenças crônicas não transmissíveis na vida do idoso acarretaria mais modificações em suas capacidades e funcionalidades.

Atualmente, sabe-se que a socialização é imprescindível para o indivíduo e que o isolamento social, principalmente emidosos, pode levar a quadros depressivos, podendo trazer várias consequências à saúde dos mesmos. Nesse contexto, a linguagem e a cognição são aspectos que merecem a preocupação de profissionais que trabalham com idosos, já que elas são essenciais para a comunicação e para o uso efetivo da língua. Assim, estudos que visem o diagnóstico e a promoção da linguagem e da cognição nesses indivíduos, são importantes para a promoção da socialização dos idosos 
Com o aumento do número de idosos, o Ministério da Saúde passou a ter um olhar diferenciado e publicou a Política Nacional de Saúde da Pessoa Idosa (PNSPI), tendo finalidade principal recuperar, manter e promover a autonomia e a independência dos indivíduos idosos, direcionando medidas coletivas e individuais de saúde para esse fim.

Para que os objetivos fossem alcançados foram definidos como diretrizes essenciais: à promoção do envelhecimento ativo e saudável; atenção integral à saúde da pessoa idosa; estímulo às ações intersetoriais; provimento de recursos capazes de assegurar qualidade da atenção à saúde da pessoa idosa; estímulo à participação e fortalecimento do controle social; formação e educação permanente dos profissionais de saúde do SUS envolvidos no cuidado ao idoso; divulgação e informação sobre a Política Nacional de Saúde da Pessoa Idosa para profissionais de saúde, gestores e usuários do SUS; promoção de cooperação nacional e internacional das experiências na atenção à saúde da pessoa idosa; e apoio ao desenvolvimento de estudos e pesquisas (BRASIL, 2006).

Como foco na PNSI, a qualidade de vida da população idosa envolve uma combinação de inúmeros fatores, alcançando desde os aspectos biológicos, funcionais até os socioculturais. Sendo assim, o envelhecimento bem-sucedido pode ser entendido como a combinação da baixa probabilidade de adoecimento e deficiências, manutenção das funções físicas e cognitivas, engajamento na vida, incluindo atividades produtivas, espiritualidade, relacionamentos interpessoais e boas condições de vida (BRAGA et al, 2011).

\section{METODOLOGIA}

Trata-se de um estudo de campo, descritivo, observacional e transversal, que foi avaliado e aprovado pelo Comitê de Ética da instituição de origem, sob o parecer de número 78707417.2.0000.5176.

A pesquisa foi realizada em uma unidade de saúde da família e em um projeto social voltado a atenção à saúde da pesso a idosa de uma capital nordestina. Participaram da pesquisa 65 idosos, de ambos os gêneros, com idades variando entre 60 e 87 anos, com média de 70,7 anos.

Como critérios elegibilidades, os participantes deveriam aceitar participar do estudo; possuir ou não hipertensão e diabetes; assinar o Termo de consentimento Livre e Esclarecido - TCLE; e não possuir alguma alteração auditiva ou neurológica que prejudicasse os procedimentos da coleta dos dados.

Para realização da pesquisa foram utilizados: 1) um questionário de informações pessoais, no qual coletamos a idade, gênero, queixas de linguagem e memória, se o participante possuía alguma doença crônica e informações médica em geral para averiguar os critérios de elegibilidade; 2) a Bateria Breve de Rastreio Cognitivo (BBRC), que foi desenvolvida pelo grupo de Neurologia Cognitiva e do 
Comportamento da Faculdade de Medicina da USP (NITRINI, et al., 2005). Este instrumento é utilizado para análise comportamental, déficit cognitivo e funcional. Consiste em um mini exame avaliando o funcionamento cognitivo global e de tarefa de fluência verbal (FV). É um instrumento de fácil aplicação e pode ser utilizado por profissionais da área da saúde, além de ser compreensível a diferentes grupos de distintas faixas de escolaridade.

Os idosos que concordaram em participar foram orientados sobre o objetivo e os procedimentos da pesquisa, concluindo com a leitura e assinatura do Termo de Consentimento Livre e Esclarecido.

Em seguida, os pesquisadores realizaram a avaliação das habilidades de memória através da BBRC (NITRINI, et al., 2005) que inclui

$\checkmark$ Teste de Nomeação e percepção visual de 10 figuras simples apresentadas em uma folha de papel, (sapato, colher, pente, árvore, tartaruga, chave, avião, casa, livro e balde) e solicitado ao indivíduo que nomeie os 10 itens.

$\checkmark$ Teste de memória incidental: após apresentação da folha com as figuras, é solicitado que o indivíduo nomeie as figuras sem o apoio visual, consentindo o tempo de um minuto para a recordação.

$\checkmark$ Avaliação da memória imediata: A folha é novamente apresentada para o indivíduo por 30 segundos. Após esse período, a folha é retirada da vista do indivíduo, em seguida é solicitado que sejam nomeadas as figuras em um prazo de um minuto.

$\checkmark$ Aprendizado é o mesmo procedimento utilizado para avaliação da memória imediata, com a orientação de que será solicitada lembrança após um intervalo.

$\checkmark$ Para avaliar a memória tardia, o indivíduo deve permanecer 5 minutos sem visualizar a folha com as 10 figuras, após esse período é solicitado uma nova nomeação das figuras. Durante esse intervalo de 5 minutos, o indivíduo é submetido à interferência com a aplicação do teste de FV para categoria de animais e do teste de desenho do relógio (TDR) com todos os números de 1 a 12 e os ponteiros mostrando um horário estabelecido. A indicação de horário utilizada no presente estudo foi "duas horas e quarenta e cinco minutos" (02:45).

$\checkmark \mathrm{Na}$ prova de FV semântica, os pesquisadores solicitavam que os idosos falassem todos os nomes de animais que eles lembravam em 60 segundos. $E$, nesse estudo, foi realizada a prova de FV fonológica, na qual os participantes foram solicitados a evocar todos os nomes que iniciavam com a letra "A" que lembravam em 60 segundos.

$\checkmark$ Por fim, os participantes deveriam visualizar uma prancha com 20 figuras e identificar quais as dez que ele tinha visualizado no início da testagem. Ressalta-se que se o participante falar um nome que não era alvo, este 
acréscimo é considerada uma intrusão e deve ser anotada pelo pesquisador em todas as provas.

Os dados coletados foram transferidos para planilha eletrônica digital para construção do banco de dados. Em seguida, submetidos a uma análise quantitativa descritiva e inferencial, a partir do software Statistical Package for Social Sciences (SPSS), versão 20.0.

A análise inferencial foi realizada para observar a correlação entre a idade e os escores obtidos nas provas de memória. Para isso, foi utilizado a Correlação de Pearson, adotando o nível de significância de 5\%.

\section{RESULTADOS}

A amostra foi composta por um total de 65 idosos, com a faixa etária entre 60 a 87 anos, sendo 46 (70,7\%) do gênero feminino e 19 (29,3\%) do gênero masculino. A maioria dos idosos apresentou DCNT, sendo a HAS a mais prevalente (Tabela 1).

Tabela 1. Características observadas nos idosos

\begin{tabular}{ccccccc}
\hline DOENÇA CRÔNICA & FEMININO & $\%$ & MASCULINO & $\%$ & Total Geral & $\%$ \\
\hline DIABETES & 4 & $6,69 \%$ & 4 & $21,05 \%$ & 8 & $12,30 \%$ \\
HIPERTENSÃO & 20 & $43,47 \%$ & 4 & $21,05 \%$ & 24 & $36,92 \%$ \\
HIPERTENSÃO + DIABETES & 9 & $19,56 \%$ & 3 & $15,78 \%$ & 12 & $18,46 \%$ \\
SEM DCNT & 13 & $28,26 \%$ & 8 & $42,10 \%$ & 21 & $32,30 \%$ \\
\hline
\end{tabular}

Legenda: DCNT = Doenças crônicas não transmissíveis

Foram observados que a maioria dos idosos $64,61 \%$ declararam queixa de memória e 83,07\% declaram não apresentar queixa de linguagem (Tabela 2).

Tabela 2. Características observadas nos idosos dos grupos do município de João Pessoa-PB, 2018

\begin{tabular}{ccccccc}
\hline QUEIXAS & FEMININO & $\%$ & MASCULINO & $\%$ & Total Geral & $\%$ \\
\hline AUSÊNCIA DE QUEIXA DE MEMÓRIA & 14 & $30,43 \%$ & 9 & $47,36 \%$ & 23 & $35,38 \%$ \\
PRESENÇA DE QUEIXA DE MEMÓRIA & 32 & $69,56 \%$ & 10 & $52,63 \%$ & 42 & $64,61 \%$ \\
AUSÊNCIA DE QUEIXA DE LINGUAGEM & 39 & $84,78 \%$ & 15 & $78,94 \%$ & 54 & $83,07 \%$ \\
PRESENÇA DE QUEIXA DE LINGUAGEM & 7 & $15,21 \%$ & 4 & $21,05 \%$ & 11 & $16,92 \%$ \\
\hline
\end{tabular}

Analisando o desempenho na avaliação, a média geral dos participantes está dentro dos padrões de normalidade nos testes, com exceção das provas de fluência verbal. Observa-se que o grupo de idosos com diabetes foi o grupo com pior desempenho na avaliação e com mais provas abaixo do padrão de normalidade, sendo: memória incidental, memória imediata, aprendizado e FV 
semântica e fonológica. Os idosos do grupo sem DCNT foram os que apresentaram o melhor desempenho na avaliação (Tabela 3).

Tabela 3. Médias geral dos idosos na avaliação de memória e média por grupos de condições de saúde.

\begin{tabular}{|c|c|c|c|c|c|c|c|c|}
\hline \multirow{2}{*}{ PROVAS } & \multicolumn{2}{|c|}{$\begin{array}{c}\text { IDOSOS COM } \\
\text { HAS }\end{array}$} & \multicolumn{2}{|c|}{$\begin{array}{l}\text { IDOSOS COM } \\
\text { DIABETES }\end{array}$} & \multicolumn{2}{|c|}{$\begin{array}{l}\text { IDOSOS SEM } \\
\text { DCNT }\end{array}$} & \multicolumn{2}{|c|}{$\begin{array}{l}\text { MÉDIA } \\
\text { GERAL }\end{array}$} \\
\hline & MÉDIA & DP & MÉDIA & $\mathrm{DP}$ & MÉDIA & DP & MÉDIA & DP \\
\hline NOMEAÇÃO & 9,9 & 0,3 & 9,6 & 0,7 & 9,9 & 0,3 & 9,8 & 0,4 \\
\hline MEMÓRIA INCIDENTAL & 5,1 & 1,9 & 4,9 & 1,1 & 5,6 & 1,7 & 5,3 & 1,7 \\
\hline MEMÓRIA IMEDIATA & 7 & 2 & 5,9 & 1,7 & 7,3 & 1,7 & 6,9 & 1,9 \\
\hline APRENDIZADO & 7,1 & 2,4 & 6,6 & 2 & 8,2 & 1,5 & 7,5 & 1,9 \\
\hline FLUÊNCIA VERBAL SEMÂNTICA & 11,1 & 3,2 & 10,6 & 4,4 & 11,9 & 3,8 & 11,1 & 3,6 \\
\hline $\begin{array}{l}\text { FLUÊNCIA VERBAL } \\
\text { FONOLOGGICA }\end{array}$ & 8,2 & 3,6 & 5,2 & 3,6 & 7,8 & 3,8 & 7,8 & 3,6 \\
\hline MEMÓRIA TARDIA & 7,2 & 3,4 & 6,2 & 2,2 & 7,6 & 1,8 & 7,1 & 2 \\
\hline RECONHECIMENTO & 9 & 1,7 & 9 & 1,3 & 9,2 & 1 & 8,9 & 1,6 \\
\hline INTRUSÕES & 0,9 & 2,2 & 1,4 & 1,8 & 0,5 & 1,4 & 0,9 & 1,9 \\
\hline
\end{tabular}

Legenda: HAS=Hipertensão Arterial Sistêmica; DCNT=Doenças crônicas não transmissíveis

Não foi identificada diferença estatística do desempenho de memória entre os grupos de idosos com e sem doenças crônicas. Contudo, foi observada correlação negativa entre a idade e o desempenho nas provas de memória imediata $\left(p=0,004^{*}\right)$, Aprendizado $\left(p=0,020^{*}\right)$, memória tardia $\left(p=0,013^{*}\right)$ e pela quantidade de Intrusões realizadas ( $p=0,006^{*}$ ). Ou seja, quanto maior a idade dos idosos, menor o desempenho nesses itens (Tabela 4).

Tabela 4. Correlação entre idade e o desempenho de memória

\begin{tabular}{ccc}
\hline Idade & Estatística do Teste & p-valor \\
\hline MEMÓRIA INCIDENTAL & $-0,19$ & 0,129 \\
MEMÓRIA IMEDIATA & $-0,354$ & $0,004^{*}$ \\
APRENDIZADO & $-0,288$ & $0,020^{*}$ \\
FLUÊNCIA VERBAL SEMÂNTICA & $-0,111$ & 0,378 \\
FLUÊNCIA VERBAL FONOLÓGICA & $-0,056$ & 0,657 \\
DESENHO DO RELÓGIO & $-0,134$ & 0,291 \\
MEMÓRIA TARDIA & $-0,31$ & $0,013^{*}$ \\
RECONHECIMENTO & $-0,198$ & 0,114 \\
INTRUSÕES & $-0,339$ & $0,006^{*}$ \\
\hline Análise inferencial: Correlação de Pearson. P-valor $=0,05^{*}$
\end{tabular}

\section{DISCUSSÃO}

Pode-se observar que, na presente pesquisa, houve a prevalência de participantes do gênero feminino (Tabela 1). A literatura tem relatado que há uma predominância de mulheres com queixas de declínio na memória em relação aos 
homens (SILVA et al., 2014; BERNARDES et al., 2016). Porém, nesse estudo, não foi possivel demonstrar diferença significante entre os gêneros, pelo fato da maioria da população estudada ser do gênero feminino. De acordo com Bernardes et al., (2016) tal fato, ocorre devido a maior sobrevida das mulheres e sua maior adesão aos grupos de idosos.

Observou-se que HAS obteve maior prevalência de DCNT (Tabela 1). Idosos hipertensos, quando comparados com idosos com pressão arterial estável, apresentam um risco maior de declínio cognitivo incluindo a demora nas respostas, memória e função executiva (ELIAS et al., 2005; ALMEIDA-PITITTO, FILHO, CENDOROGLO, 2008; MATOSO et al., 2013). Estudo objetivou comparar o desempenho cognitivo entre idosos normotensos e idosos hipertensos, por meio de testes diferentes de avaliação neuropsicológica, e observou que em todos os testes os idosos hipertensos tiveram desempenho cognitivo inferior quando relacionados aos normotensos (MATOSO et al., 2013).

As alterações de memória na senescência representam uma das queixas mais frequentes nos idosos (ROGRIGUES, 2014). Como foi possível observar nesta pesquisa, que os idosos apresentaram mais queixas de memória do que de linguagem (Tabela 2). Em alguns casos, o declínio cognitivo evolui para a demência, confirmando que as queixas de memória podem ser estimadas parte do conceito de metamemória, ou seja, percepções e autoavaliações da memória (YASSUDA, et al., 2005; MOTA et al., 2008; BERNARDES et al., 2016).

Foi possível observar que o grupo de idosos com diabetes apresentou maior risco para demência do que os outros grupos (Tabela 3). O diabetes tem sido associado a um aumento de risco para quedas, depressão, declínio físico e cognitivo (ALMEIDA-PITITTO; FILHO; CENDOROGLO, 2008).

Alguns mecanismos fisiopatológicos vêm sendo estudados, como elo de ligação entre alterações metabólicas do diabetes e disfunção cognitiva, como alterações vasculares, acúmulo de $\beta$-amilóide intracerebral, alteração de estruturas cerebrais e processo inflamatório que levam à lesão neuronal (ALMEIDA-PITITTO; FILHO; CENDOROGLO, 2008). Outro estudo refere que o DM, pode aumentar a ação do sistema imunológico, levando a liberação de moléculas de citocinas próinflamatórias, o que pode contribuir para a neuroinflamação do cérebro, sendo essa característica fisiopatológica da doença de Alzheimer (NAZARETH, 2017).

Além disso, os idosos sem DCNT foram os que apresentaram um melhor desempenho no teste de memória (Tabela 3), indicando que o desenvolvimento de políticas públicas voltadas à prevenção de DCNT favorece a manutenção das funções cognitivas no envelhecimento.

Pode-se observar nos resultados estatísticos que não houve diferença no desempenho de memória em relação com os grupos de idosos com e sem doenças crônicas. Porém foi observado que quanto maior a idade dos idosos menor foram o desempenho nas provas de memória imediata, aprendizado e pela quantidade 
de Intrusões realizadas. Ou seja, quanto maior o avanço da idade, maior o declínio cognitivo (Tabela 4), contribuindo com o estudo de Nascimento et al., (2015) que objetivou averiguar a prevalência e os fatores associados ao declínio cognitivo em idosos, e observou que os indivíduos mais velhos (40\%), com faixa etária $\geq 80$ anos e escolaridade menor, tiveram estatisticamente maior comprometimento a nível cognitivo.

A preservação de conhecimentos adquiridos ao longo da vida é mantida até os 70 anos, após essa idade, existem reduções nas habilidades executivas. Tais reduções manifestam-se com a diminuição na atenção, memória imediata e da capacidade de planejamento antecipatório das ações, que independem de sexo e das condições socioeconômicas (VALE et al., 2008). O envelhecimento normal é caracterizado pelo declínio cognitivo da memória, função executiva e habilidades que envolvem velocidade, iniciando-se na fase de meia idade e prosperam até o óbito (PARENTE, 2006).

Visando retardar o declínio cognitivo, fazem-se necessárias ações de promoção e prevenção de agravos na saúde do idoso. A comunicação é um dos principais meios de interação do indivíduo desse público. Com isso, o fonoaudiólogo desenvolverá ações estimulando e eliminando os fatores que comprometam essa habilidade, buscando adaptar as necessidades dessa população.

A Fonoaudiologia tem expandido seu campo de trabalho e áreas de atuação, junto à atenção á saúde do idoso, atuando em pesquisas, prevenção, avaliação, diagnóstico, terapia, orientação e promoção de saúde. Além de aprimoramento nas áreas da Linguagem, Voz, Audiologia, Motricidade Orofacial e Saúde Coletiva (MINISTÉRIO DA SAÚDE, 2006).

Em específica, a atuação na Saúde Coletiva, o fonoaudiólogo incrementará a importância da promoção da saúde, da prevenção de riscos e agravos, da reorientação da assistência aos idosos, e da qualidade de vida, privilegiando modificações nos estilos de vida e nas relações sociais entre todos os sujeitos envolvidos no cuidado (NERI, 2001).

No que diz respeito às dificuldades nas atividades de vida diárias (AVDs) do idoso o declínio cognitivo repercute na limitação da realização de tarefas básicas, ocasionando problemas no convívio social que podem vir a causar depressão e isolamento (RAMOS, 2003).

Portanto, a estimulação cognitiva dá se por meio de recursos lúdicos, técnicas de memorização, e adaptações de materiais da rotina baseando se na necessidade individual do idoso, favorecendo assim, os atos da vida cotidiana. Para tanto é essencial apoio psicológico e promover a socialização (CAMARA et al., 2009). 


\section{CONSIDERAÇÕES FINAIS}

Verificou-se que não houve diferença estatística do desempenho de memória em relação aos grupos de idosos com e sem doenças crônicas. Porém, foi observado que o declínio cognitivo de memória em idosos aumenta de acordo com o avanço da idade. As queixas de memória são maiores do que as queixas de linguagem nos participantes da pesquisa. E os participantes com diabetes foram os que tiveram um maior declínio cognitivo no rastreio realizado.

Um bom funcionamento das funções cognitivas favorece a qualidade de vida no envelhecimento. Para isso, é importante que os profissionais saúde, em conjunto com a população, desenvolvam estratégias de prevenção de alterações cognitivas e promoção de um envelhecimento saudável e ativo, a fim de minimizar as alterações do declínio cognitivo na população idosa.

\section{REFERENCIAS}

ABREU, I.D.; FORLENZA, O.V.; BARROS, H.L. Demência de Alzheimer: correlação entre me-mória e autonomia. Revista de Psiquiatria Clínica, São Paulo, vol.32, n.3, p.131136, 2005.

ALMEIDA-PITITTO, Bianca; FILHO, Clineu de M. Almada; CENDOROGLO, Maysa S. Déficit cognitivo: mais uma complicação do diabetes melito? Arquivos Brasileiros de Endocrinologia Metabólica, São Paulo, v. 52, n. 7, p. 1076-1083, Outubro. 2008.

ALVARENGA, Patrícia Perreira; PEREIRA, Daniele; ANJOS, Daniela Maria da Cruz. Mobilidade funcional e função executiva em idosos diabéticos e não diabéticos. Revista Brasileira de Fisioterapia, São Carlos, v. 14, n. 6, p. 491-496, 2010.

ARAKI, Atsushi; ITO, Hideki. Diabetes mellitus and geriatric syndromes. Geriatrics \& gerontology international, Tokyo, v. 9, n. 2, p. 105-114, 2009.

BADDELEY, Alan D. Is working memory still working? European Psychologist, Kirkland, v.7, n 2, p. 85-97, Junho. 2002.

BERNARDES, Flavia Rodrigues et al. Queixa subjetiva de memória e a relação com a fluência verbal em idosos ativos. CoDAS, São Paulo, v. 29, n. 3, p. e20160109, 2016

BRAGA, S.F.M.; et al. Fatores associados com a QV relacionada à saúde de idosos em HD. Revista Saúde Pública, v.45, n.6, P.1127-1136, 2011.

BRASIL. Envelhecimento e Saúde da Pessoa Idosa. Brasília: Ministério da Saúde; 2006. $192 p$. 
BRASIL. Ministério da Saúde. PORTARIA N² 2.528 DE 19 DE OUTUBRO DE 2006. Aprova a Política Nacional de Saúde da Pessoa Idosa. 2006.

BRASIL. MINISTÉRIO DA SAÚDE. Portal da Saúde. Disponível em:<http://portalsaude. saude.gov.br/index.php/cidadao/principal/agencia-saude/25924-ministeriorecomenda-e-preciso-envelhecer-com-saude>. Acesso em: 02 março de 2018.

CAMARA, Vilma Duarte et al. Reabilitação Cognitiva das Demências. Revista Brasileira de Neurologia, Rio de Janeiro, v.45, n.1, p. 25-33, 2009.

CAMPOLINA, Alessandro Gonçalves. A transição de saúde e as mudanças na expectativa de vida saudável da população idosa: possíveis impactos da prevenção de doenças crônicas. Cadernos de Saúde Pública, Rio de Janeiro, v. 29, n. 6, p. 1217 1229, 2013.

CAMPOLINA, Alessandro Gonçalves; DINI, Patrícia Skolaude; CICONELLI, Rozana Mesquita. Impacto da doença crônica na qualidade de vida de idosos da comunidade em São Paulo (SP, Brasil). Ciência e Saúde Coletiva, Rio de Janeiro, v. 15, n. 6, p. 2919-2925, 2011.

CEMBRANEL, Francieli et al. Impacto do diagnóstico de diabetes e/ou hipertensão sobre indicadores de consumo alimentar saudável: estudo longitudinal com idosos.

Revista Brasileira de Geriatria e Gerontologia, Rio de Janeiro, v. 20, n. 1, p. 34-36, 2017.

ELIAS, Merrill F et al. Untreated blood pressure level is inversely related to cognitive functioning: the Framingham Study. American Journal of Psychiatry, Arlington, v. 138, n.6, p. 353-64, 1993.

IRIGARAY, Tatiana Quarti; FILHO, Irenio Filho; SCHNEIDER, Rodolfo Herberto. Efeitos de um treino de atenção, memória e funções executivas na cognição de idosos saudáveis. Psicologia: Reflexão e Critica, Porto Alegre, v. 25, n. 1, p. 182-187, 2012.

IRIGARAY, Tatiana Quarti; SCHNEIDER, Rodolfo Herberto; GOMES, Irenio. Efeitos de um treino cognitivo na qualidade de vida e no bem-estar psicológico de idosos. Psicologia: Reflexão e Crítica, Porto Alegre, v. 24, n. 4, p. 810-8, 2011.

MAC-KAY, A.P.M.G. Afasias e Demências: avaliação e tratamento fonoaudiológico. São Paulo: Santos, 2011.

MATOSO, Juliana Magalhães Duarte et al. Idosos hipertensos apresentam menor desempenho cognitivo do que idosos normotensos. Arquivos Brasileiros de Cardiologia, São Paulo, v. 100, n. 5, p. 444-451, 2013. 
MAZO, Giovana Zarpellon et al. condições de saúde, a análise de quedas e o nível de atividade física dos idosos. Revista Brasileira de Fisioterapia, São Carlos, v. 1 1, n. 6 , p. 437-442, 2007.

MOTA, Márcia Maria Peruzzi Elia da et al . Triagem cognitiva: comparações entre o mini-mental e o teste de trilhas. Estudos de Psicologia, Campinas, v.25, n. 3, p. 353359, Setembro. 2008.

MUNSHI, Medha et al. Cognitive Dysfunction Isassociated With Poor Diabetes Control In Older Adults. Diabetes Care, Alexandria, v. 29, n. 8, p. 1794-1799, 2006.

MURRAY, Michael D et al. Preservation of cognitive function with antyhipertensive medications: a longitudinal analysis of community-based sample of African Americans. Archives of internal medicine, Chicago, v. 162, n. 18, p. 2090-2096.

NAZARETH, Aparecida Marcelino. Type 2 diabetes mellitus in the pathophysiology of Alzheimer's disease. Dementia \& neuropsychologia, São Paulo, v. 11, n. 2, p. 105-113, 2017.

NERI, Anita Liberalesso. Envelhecimento e qualidade de vida na mulher. In: Congresso Paulista De Geriatria E Gerontologia, 2., 2001, São Paulo. São Paulo: GERP, 2001, p. 01-18.

NITRINI, Ricardo et al . Diagnóstico de doença de Alzheimer no Brasil: avaliação cognitiva e funcional. Recomendações do Departamento Científico de Neurologia Cognitiva e do Envelhecimento da Academia Brasileira de Neurologia. Arquivos de Neuro-Psiquiatria, São Paulo, v. 63, n. 3a, p. 720-727, Setembro. 2005.

PAPADEMETRIOU, Vasilios. Blood pressure regulation and cognitive function: A review of the literature. Geriatrics, New York, v. 60, n.1, p.20-4, 2005.

PARENTE, Maria Alice de Mattos Pimenta. Cognição e Envelhecimento. Porto Alegre: ArtMed; 2006.

RAMOS, Luiz Roberto. Fatores determinantes do envelhecimento saudável em idosos residentes em centro urbano: Projeto Epidoso, São Paulo. Cadernos de Saúde Pública, Rio de Janeiro, v. 19, n. 3, p. 793-797, Junho. 2003.

RODRIGUES, Flávia de Sousa. Declínio Cognitivo na Senescência e Repercussão no Contexto Familiar. Monografia (Licenciatura em Psicologia)- Faculdade de Psicologia- Universidade Católica Portuguesa. 2014. 
RYAN, Christopher M., GEKCLE, Michelle O. Circumscribed Cognitive Dysfunction In Middle-Aged Adults With Type 2 Diabetes. Diabetes Care, Alexandria, v. 23, n. 10, p. 1486-1493, 2000.

SILVA, Kelly da et al. Caracterização da memória de adultos e idosos hospitalizados. Distúrbios da Comunicação, São Paulo, v. 29, n.2, p. 218-26, 2017.

SILVA, Lais Dos Santos Vinholi; et al . Relations between memory complaints, depressive symptoms and cognitive performance among community dwelling elderly. Revista de Psiquiatria Clínica, São Paulo, v. 41, n. 3, p. 67-71, 2014.

VALE, Tacianny Lorena Freitas do et al . Envelhecimento e memória episódica: desempenho de 15 idosos no BVMT-R e HVLT-R. Psicologia hospitalar, (São Paulo), São Paulo, v. 6, n. 2, p. 74-87, Junho, 2008.

YASSUDA, Mônica Sanches et al. Um Estudo de Validação de Instrumentos de Pesquisa sobre Memória e Envelhecimento. Psicologia: Reflexão e Crítica, Porto Alegre, v. 18, n. 1, p. 78-90, 2005. 